\title{
Physiological Function of Mycobacterial mtFabD, an Essential Malonyl-CoA:AcpM Transacylase of Type 2 Fatty Acid Synthase FASII, in Yeast mct1 $\Delta$ Cells
}

\author{
Aner Gurvitz \\ Section of Physiology of Lipid Metabolism, Center for Physiology, Pathophysiology and Immunology, Institute of Physiology, \\ Medical University of Vienna, Schwarzspanierstrasse 17, 1090 Vienna, Austria \\ Correspondence should be addressed to Aner Gurvitz, aner.gurvitz@meduniwien.ac.at
}

Received 16 March 2009; Accepted 31 July 2009

Recommended by Eivind Hovig

Mycobacterium tuberculosis mtFabD is an essential malonyl-CoA:AcpM transacylase and is important for vital protein-protein interactions within type 2 fatty acid synthase FASII. mtFabD contacts KasA, KasB, FabH, InhA, and possibly also HadAB, HadBC, and FabG1/MabA. Disruption of mtFabD's interactions during FASII has been proposed for drug development. Here, the gene for a mitochondrially targeted mtFabD was ectopically expressed in Saccharomyces cerevisiae mct1 $\Delta$ mutant cells lacking the corresponding mitochondrial malonyl-CoA transferase Mct1p, allowing the mutants to recover their abilities to respire on glycerol and synthesize lipoic acid. Hence, mtFabD could physiologically function in an environment lacking holo-AcpM or other native interaction partners.

Copyright () 2009 Aner Gurvitz. This is an open access article distributed under the Creative Commons Attribution License, which permits unrestricted use, distribution, and reproduction in any medium, provided the original work is properly cited.

\section{Introduction}

A new approach for eradicating tuberculosis has been proposed that centers on perturbing vital protein-protein interactions associated with the essential mycobacterial process of type 2 fatty acid synthase, FASII [1]. FASII is dedicated to the production of mycolic acids and is targeted by isoniazid, which represents only one of less than a handful of veteran first-line drugs for combating Mycobacterium tuberculosis. Mycolic acids are very long chain $\alpha$-branched $\beta$-hydroxylated fatty acids that help form the pathogen's defensive layer [2].

A critical step in mycobacterial FASII is represented by the transfer of the malonyl moiety from coenzyme A (CoA) to acyl carrier protein (activated holo-AcpM), which is effected by a malonyl-CoA:AcpM transacylase mtFabD (Rv2243), so as to generate malonyl-AcpM [3]. Importantly, $\mathrm{mtFabD}$ also plays a key role in holding together FASII enzymes within a protein complex [1]. The structure of this complex is presumed to occur as a constellation of a core triad, with each one of the three identical core units independently contacting a single condensing enzyme, KasA,
$\mathrm{KasB}$, or FabH. KasB is further linked to Pks13 responsible for subsequent maturation of the nascent mycolic acid, and both KasA and KasB provide condensing activities for additional methyltransferases during this process [1].

The FASII core unit [1] is thought to be composed of mtFabD, FabG1/MabA (representing 3-oxoacyl-AcpM reductase), InhA (2-trans-enoyl-AcpM reductase), and 3hydroxyacyl-AcpM dehydratase, ostensibly HadAB and HadBC [4]. Within this core, mtFabD occurs in close proximity to-and also forms specific protein-protein interactions with-itself, InhA, and possibly also with $\mathrm{HadAB}$ and HadBC. In addition, it can also be directed to contact mutant versions of nondimerizing FabG1/MabA [1]. It was proposed that disrupting mtFabD interactions may be detrimental for mycobacterial survival, and this should be considered as a possible new approach in the search for novel antituberculous drugs [1]. Hence, it would be important to study $\mathrm{mtFabD}$ function in a situation where these formal protein-protein interactions are altered or nonexistent, such as in yeast mitochondrial FASII.

The fungal FASII equivalent of mtFabD is exemplified by Saccharomyces cerevisiae Mctlp [5]. Mctlp is structurally 


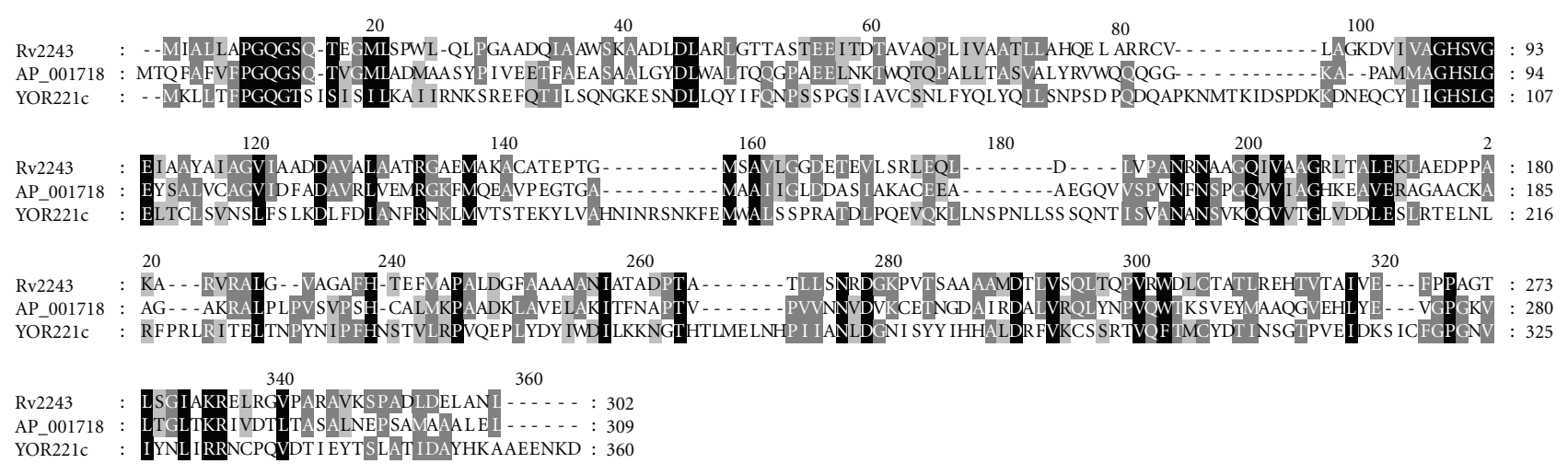

Figure 1: Comparison of M. tuberculosis mtFabD with its E. coli and S. cerevisiae homologs. Multalin- and Genedoc-based comparison of the deduced amino acid sequence of M. tuberculosis FabD (Rv2243), E. coli FabD (AP_001718), and S. cerevisiae Mctlp (Yor221cp). Dashes indicate the arrangement of the sequences for best fit. Black shadings refer to conserved amino acid residues among all three sequences whereas the darker and lighter grey shadings denote regions with more relaxed residue similarities not necessarily shared by the full set of sequences.

more distant to the mycobacterial protein than is Escherichia coli FabD (Figure 1), whose latter temperature-dependent inactivation can be compensated for by ectopically expressing mtFabD [3]. Yeast mutant cells devoid of Mctlp contain underdeveloped mitochondria, fail to respire or produce sufficient levels of lipoic acid, and are exclusively fermentative [5]. Here, mutant yeast cells expressing a mitochondrially targeted form of mycobacterial mtFabD were compared to an otherwise isogenic wild-type strain for growth, respiration, and lipoic acid production, and the implications are discussed.

\section{Materials and Methods}

The $f a b D$ sequence was amplified from $\mathrm{H} 37 \mathrm{Rv}$ genomic DNA by thermocycling using oligonucleotides MLS-FabD F $5^{\prime}$-TTATCCATGGTTGCGTTGCTCGCACCCGGAC-3' and FabD R $5^{\prime}$-TATTAAGCTTATAGGTTTGCCAGCTCGTCC$3^{\prime}$ that introduced $5^{\prime} \mathrm{NcoI}$ and $3^{\prime} \mathrm{HindIII}$ sites. The amplified $f a b D$ DNA was processed in such a way that it was preceded by the nucleotides for the Coq3p [6] mitochondrial leader sequence (MLS), and the gene fusion was ligated behind the CTA1 promoter, as described [7]. The final mtFabD expression plasmid, referred to as pYE352:CTA1-COQ3FABD, was based on a URA3-marked YEp352 multicopy plasmid [8]. A control URA3-marked multicopy plasmid vector YEplac195 [9] was used to transform the wild type or the otherwise isogenic mutant to uracil prototrophy. Nucleotide sequencing of the $f a b D$ insert verified that no mutations were introduced during the amplification process and that the COQ3-fabD junction remained intact.

The wild-type yeast strain BY4741 (MATa his3 $\Delta 1$ leu $2 \Delta 0$ met $15 \Delta 0$ ura $3 \Delta 0$ ) and its $m c t 1 \Delta$ derivative (yor $221 c:$ : kanMX) were obtained from EUROSCARF (http://www .uni-frankfurt.de/). Transformation of yeast strains was performed using a published method [10], and transformants were selected on solid synthetic definedglucose medium lacking uracil (SD-Ura) that consisted of $0.67 \%$ (wt/vol) yeast nitrogen base without amino acids,
2\% (wt/vol) D-glucose, and 3\% (wt/vol) agar, with all supplements added except for uracil (Sigma-Aldrich Inc. St. Louis, MO). Synthetic complete glycerol medium (SCglycerol) was made up essentially as above, but with the addition of uracil and the replacement of glucose with $3 \%(\mathrm{wt} / \mathrm{vol})$ glycerol as the sole carbon source. Other standard yeast [11] and E. coli [12] media used are described. Respiration competence was assayed by overlaying cells grown on solid SD-Ura medium with $0.1 \%$ (wt/vol) 2,3,5-triphenyltetrazolium chloride (TTC) in $0.067 \mathrm{M}$ phosphate-buffered saline and $1.5 \%(\mathrm{wt} / \mathrm{vol})$ low-melting temperature agarose [13]. Lipoic acid content of yeast strains was monitored by a biological assay described previously $[14,15]$ using the lipoic acid deficient $E$. coli strain JRG33. Multalin (http://npsa-pbil.ibcp.fr/cgibin/npsa_automat.pl?page=/NPSA/npsa_multalin.html) and Genedoc (http://www.nrbsc.org/gfx/genedoc/index.html) were accessed to construct the amino acid alignment in Figure 1.

\section{Results}

To examine whether mtFabD would be able to act as a physiological malonyl-CoA transferase in S. cerevisiae, the corresponding gene was expressed in yeast $m c t 1 \Delta$ cells devoid of the native mitochondrial malonyl-CoA:Acp1p transferase Mctlp. The mycobacterial protein was generated as a hybrid construct that was preceded by the yeast Coq3p MLS capable of directing proteins to the mitochondria [6]. As controls, mct $1 \Delta$ cells and the corresponding wild type were transformed with a URA3-marked YEplac195 plasmid vector [9]. Thereafter, the three strains were propagated overnight on SD-Ura glucose medium selecting for plasmid presence. Following tenfold serial dilution, cultures were spotted onto solid SD-Ura or SCglycerol media, and the plates were incubated at $30^{\circ} \mathrm{C}$ until single colonies were detectable. The results demonstrated that $m c t 1 \Delta$ mutants expressing $\mathrm{mtFabD}$ resembled to an extent the wild-type strain in that they were capable of some growth on glycerol as the sole 
carbon source (Figure 2a). As anticipated, those mutant cells harboring a URA3-marked plasmid vector that was devoid of a sequence for transferase activity were not able to grow or divide on this medium, albeit they demonstrated ample fermentative growth on glucose (Figure 2b). To investigate whether the growth observed for mtFabD-expressing mutant cells correlated with a regenerated electron transfer chain, 2,4,5-triphenyltetrazolium chloride (TTC) was applied to the previous SD-Ura plate. The results demonstrated that mutant $m c t 1 \Delta$ cells expressing $\mathrm{mtFabD}$ were similar to the wild type in that they generated the red chromophore (Figure 2c). Hence, mtFabD effected the recuperation of the electron transfer chain in mutant $m c t 1 \Delta$ cells, which enabled them to resume respiratory growth on a nonfermentable carbon source.

Finally, to place mtFabD action within the context of fatty acid biosynthesis in yeast mitochondria, lipoic acid production was examined in the three strains. In this test, lipoic acid was prepared from triplicate yeast cultures grown on SD-Ura, and the extracts were used to supplement a synthetic growth medium into which were inoculated auxotrophic bacterial cells. The values were calculated based on a standard curve that plotted bacterial growths versus known amounts of lipoic acid that had been added to the otherwise restrictive medium. The results showed that extracts derived from the wild-type strain led to a level of bacterial growth that was equivalent to $208 \pm 67 \mathrm{ng}$ lipoic acid per gram wet weight of yeast cells (values are mean \pm S.D., $n=3$ ), whereas those derived from mct1 $\Delta$ mutant cells harboring the plasmid vector supported only a nominal growth commensurate with 20 ng lipoic acid per gram wet weight. Importantly, extracts from mutant cells expressing mtFabD yielded near wild-type bacterial growth levels of 184 $\pm 37 \mathrm{ng}$ lipoic acid per gram wet weight. Taken together, ectopically expressed mtFabD could compensate for the loss of fungal Mctlp, and the significance of this result is discussed.

\section{Discussion}

Mycobacterium tuberculosis causes widespread human death and immense suffering, and it is estimated that about 2 million people die annually from tuberculosis. No less than one third of humanity is thought to be burdened with M. tuberculosis, which kills more adults than any other disease due to a single infectious agent [2]. Numerous tuberculosis patients are now infected with resistant strains, and so the development of novel therapeutics against $M$. tuberculosis is a pressing issue. Recently, a new approach for battling tuberculosis has been proposed, in which mtFabDdependent protein-protein interactions considered pivotal for the production of mycolic acids by FASII are to be singled out for perturbance [1].

In this study, M. tuberculosis fabD was shown to be interchangeable with the $S$. cerevisiae gene for Mctlp during respiratory growth and de novo lipoic acid biosynthesis. The chemistry underlying the present phenotype rescue of a yeast FASII mutant is fundamentally different compared

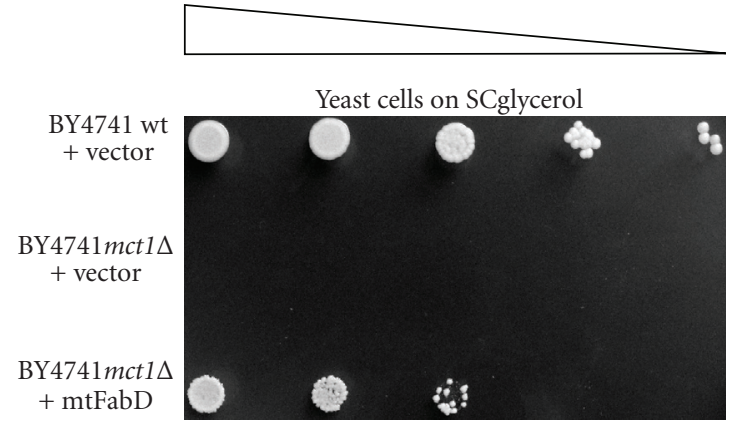

(a)

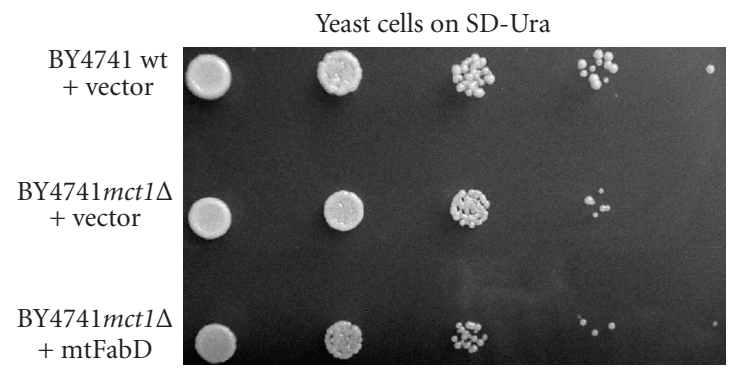

(b)

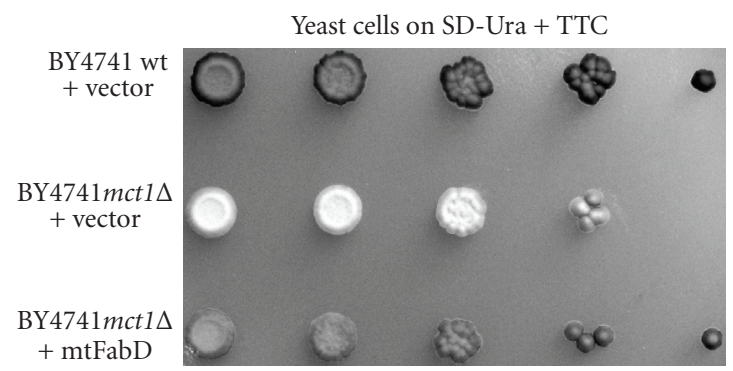

(c)

Figure 2: Respiratory growth of $S$. cerevisiae mct1 $1 \Delta$ mutant cells expressing $M$. tuberculosis mtFabD. Wild-type yeast cells harboring a YEplac195 plasmid vector and $m c t 1 \Delta$ cells similarly transformed to uracil prototrophy or expressing mitochondrially targeted Rv2243 (mtFabD) from a YEp352 plasmid (both plasmid types are marked with URA3) were grown on SD-Ura medium that selected for plasmid presence, and following serial dilution (triangle), were applied to solid media that consisted of (a) 3\% (wt/vol) glycerol (SCglycerol) or (b) $2 \%$ (wt/vol) glucose (SD-Ura). The plates were incubated at $30^{\circ} \mathrm{C}$ until single colonies appeared, and recorded photographically. For (c), following an additional $4 \mathrm{~d}$ incubation, the SD-Ura plate was overlaid with $0.1 \%$ (wt/vol) $2,4,5$ triphenyltetrazolium chloride (TTC), and the development of the red chromophore was monitored.

to previous demonstrations of physiological function within this context, for example, using InhA [7], HadAB and HadBC [16], or FabG1/MabA and FabG4 [17]. This difference lies in the fact that here mtFabD modified the thioester bond to the $4^{\prime}$-phosphopantetheine group of yeast acyl carrier protein Acp1p, whereas the previously tested enzymes acted on Acplp's cargo acyl group. There are a number of surprises associated with the present finding. One, yeast cells were capable of metabolizing very GC-rich genetic 
information $(67.3 \%$ for fabD compared with only $37.6 \%$ for MCT1) and were successful in expressing a mitochondrially targeted heterologous protein that could fold into its native conformation in a wholly nonmycobacterial environment. Two, despite mtFabD's marked preference for mycobacterial AcpM over bacterial ACP [3], it nevertheless tolerated the evolutionarily more distant yeast Acplp. And three, although mtFabD interacts within its native environment with additional FASII enzymes [1, 18], the mycobacterial transacylase was still functional in the absence of these interaction partners.

It should be underscored that the existence per se of an FASII complex in mycobacteria is not disputed as a result of this study, nor are the contacts made by mtFabD with its partners. Nevertheless, the recent demonstration of FabG1/MabA function in fungi [17] has put into question the extent to which protein contacts are really important for mycobacterial FASII [18]. The present finding that mtFabD can also work in yeast should therefore raise the alarm for those subscribing to the point of view that disrupting FASIIdependent associations would be lethal to M. tuberculosis and perhaps dampen some of their expectations that compounds capable of annulling these contacts would have pharmacological uses. The work outlined here additionally underscores the versatility of this fungal system for exploring the function of pathogen proteins in complete quarantine from their native metabolic networks.

\section{Acknowledgments}

Johanna Mäkinen from the Mycobacterial Reference Laboratory at the National Public Health Institute in Turku, Finland, is thanked for providing M. tuberculosis genomic DNA, and Katri Näppä, Alexander Kastaniotis, and J. Kalervo Hiltunen (all from the University of Oulu, Finland) for the lipoic acid measurements as well as invaluable advice and assistance. This work was supported by the Grant P20764B03 from the Austrian Science Fund (FWF).

\section{References}

[1] R. Veyron-Churlet, S. Bigot, O. Guerrini, et al., "The biosynthesis of mycolic acids in Mycobacterium tuberculosis relies on multiple specialized elongation complexes interconnected by specific protein-protein interactions," Journal of Molecular Biology, vol. 353, pp. 847-858, 2005.

[2] K. Takayama, C. Wang, and G. S. Besra, "Pathway to synthesis and processing of mycolic acids in Mycobacterium tuberculosis," Clinical Microbiology Reviews, vol. 18, pp. 81-101, 2005.

[3] L. Kremer, et al., "Biochemical characterization of acyl carrier protein (AcpM) and malonyl-CoA: AcpM transacylase (mtFabD), two major components of Mycobacterium tuberculosis fatty acid synthase II," The Journal of Biological Chemistry, vol. 276, pp. 27967-27974, 2001.

[4] E. Sacco, A. S. Covarrubias, H. M. O'Hare, et al., “The missing piece of the type II fatty acid synthase system from Mycobacterium tuberculosis," Proceedings of the National Academy of Sciences of the United States of America, vol. 104, pp. 14628-14633, 2007.
[5] R. Schneider, B. Brors, F. Bürger, S. Camrath, and H. Weiss, "Two genes of the putative mitochondrial fatty acid synthase in the genome of Saccharomyces cerevisiae," Current Genetics, vol. 32, pp. 384-388, 1997.

[6] A. Y. Hsu, W. W. Poon, J. A. Shepherd, D. C. Myles, and C. F. Clarke, "Complementation of coq3 mutant yeast by mitochondrial targeting of the Escherichia coli UbiG polypeptide: evidence that UbiG catalyzes both $O$-methylation steps in ubiquinone biosynthesis," Biochemistry, vol. 35, pp. 97979806, 1996.

[7] A. Gurvitz, J. K. Hiltunen, and A. J. Kastaniotis, "Function of heterologous Mycobacterium tuberculosis In, a type 2 fatty acid synthase enzyme involved in extending $\mathrm{C}_{20}$ fatty acids to $\mathrm{C}_{60}$ to- $\mathrm{C}_{90}$ mycolic acids, during de novo lipoic acid synthesis in Saccharomyces cerevisiae," Applied and Environmental Microbiology, vol. 74, pp. 5078-5085, 2008.

[8] J. E. Hill, A. M. Myers, T. J. Koerner, and A. Tzagoloff, "Yeast/E.coli shuttle vectors with multiple unique restriction sites," Yeast, vol. 2, pp. 163-167, 1986.

[9] R. D. Gietz and A. Sugino, "New yeast-Escherichia coli shuttle vectors constructed with in vitro mutagenized yeast genes lacking six-base pair restriction sites," Gene, vol. 74, pp. 527534, 1988 .

[10] D. C. Chen, B. C. Yang, and T. T. Kuo, "One-step transformation of yeast in stationary phase," Current Genetics, vol. 21, pp. 83-84, 1992.

[11] M. D. Rose, F. Winston, and P. Heiter, Methods in Yeast Genetics: A Laboratory Manual, Cold Spring Harbor Laboratory Press, Cold Spring Harbor, NY, USA, 1990.

[12] J. Sambrook, E. F. Fritsch, and T. Maniatis, Molecular Cloning: A Laboratory Manual, Cold Spring Harbor Laboratory Press, Cold Spring Harbor, NY, USA, 1989.

[13] E. Böker-Schmitt, S. Francisci, and R. J. Schweyen, "Mutations releasing mitochondrial biogenesis from glucose repression in Saccharomyces cerevisiae," Journal of Bacteriology, vol. 151, pp. 303-310, 1982.

[14] M. A. Hayden, I. Y. Huang, G. Iliopoulos, M. Orozco, and G. W. Ashley, "Biosynthesis of lipoic acid: characterization of the lipoic acid auxotrophs Escherichia coli W1485-lip2 and JRG33lip9," Biochemistry, vol. 32, pp. 3778-3782, 1993.

[15] S. Brody, C. Oh, U. Hoja, and E. Schweizer, "Mitochondrial acyl carrier protein is involved in lipoic acid synthesis in Saccharomyces cerevisiae," FEBS Letters, vol. 408, pp. 217-220, 1997.

[16] A. Gurvitz, J. K. Hiltunen, and A. J. Kastaniotis, “Heterologous expression of mycobacterial proteins in Saccharomyces cerevisiae reveals two physiologically functional 3-hydroxyacylthioester dehydratases, HtdX and HtdY, in addition to HadABC and HtdZ," Journal of Bacteriology, vol. 191, pp. 26832690, 2009.

[17] A. Gurvitz, "The essential mycobacterial genes, fabG1 and fabG4, encode 3-oxoacyl-thioester reductases that are functional in yeast mitochondrial fatty acid synthase type 2," Molecular Genetics and Genomics, vol. 282, pp. 407-416, 2009.

[18] R. Veyron-Churlet, O. Guerrini, L. Mourey, M. Daffé, and D. Zerbib, "Protein-protein interactions within the fatty acid synthase-II system of Mycobacterium tuberculosis are essential for mycobacterial viability," Molecular Microbiology, vol. 54, pp. 1161-1172, 2004. 

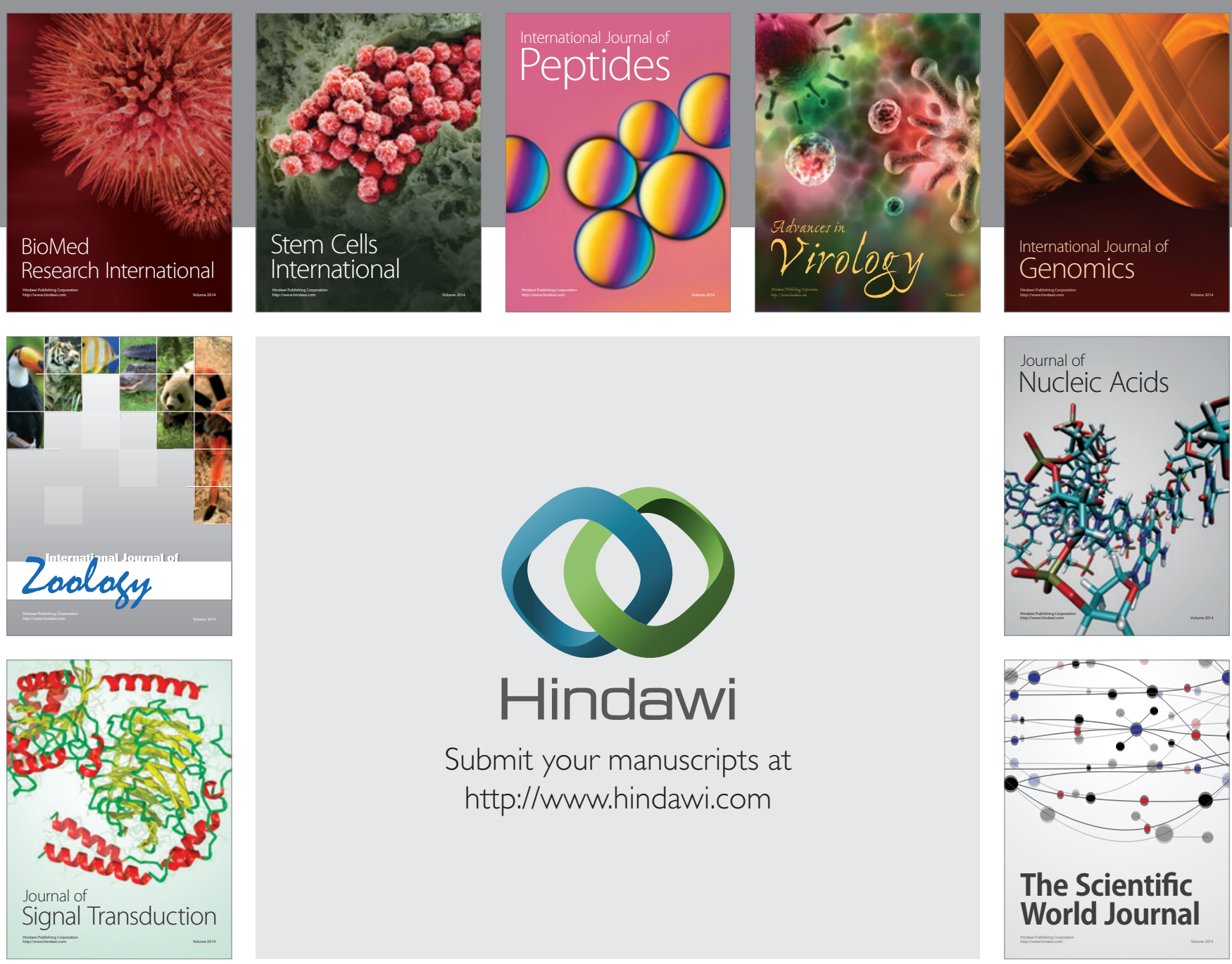

Submit your manuscripts at

http://www.hindawi.com
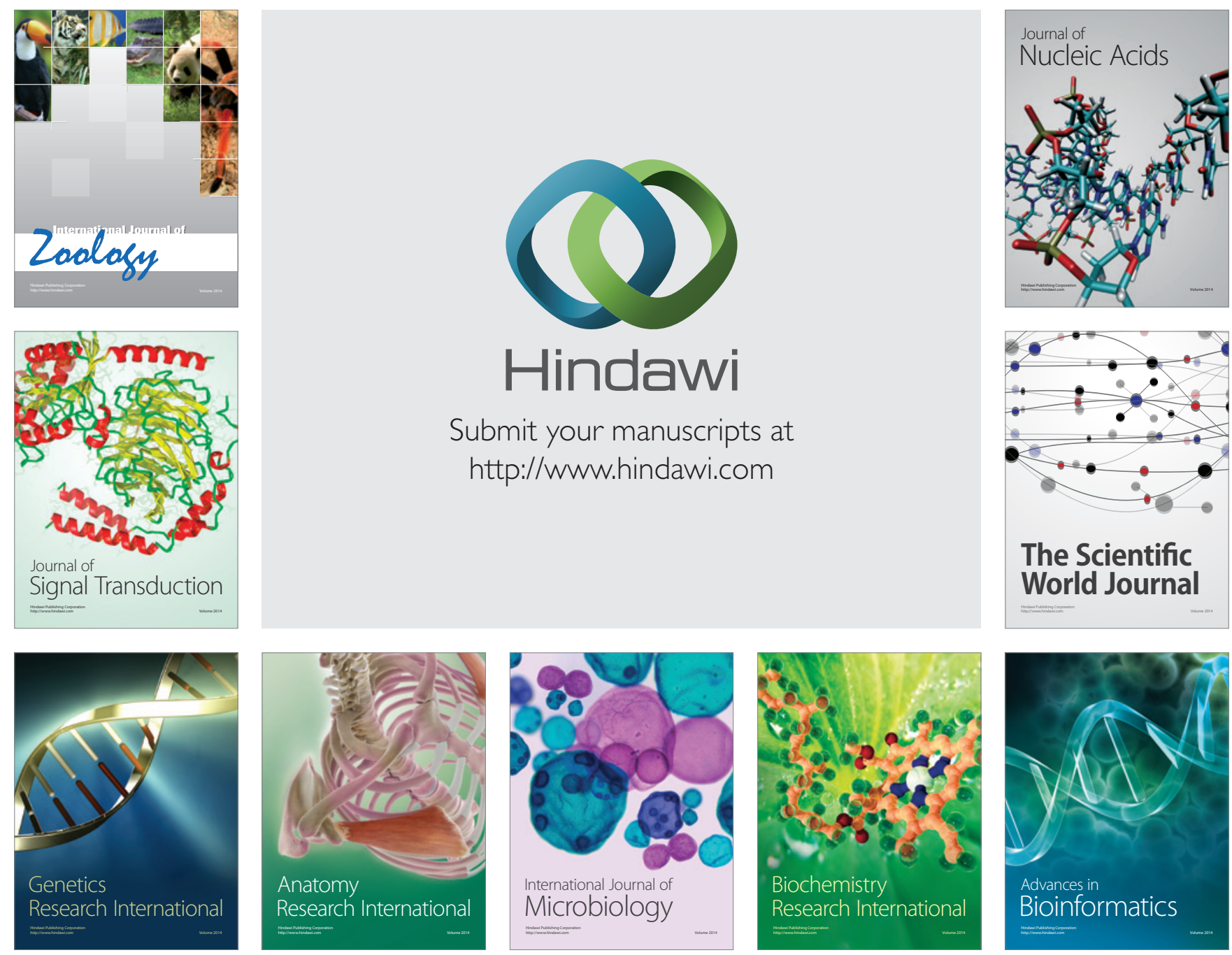

The Scientific World Journal
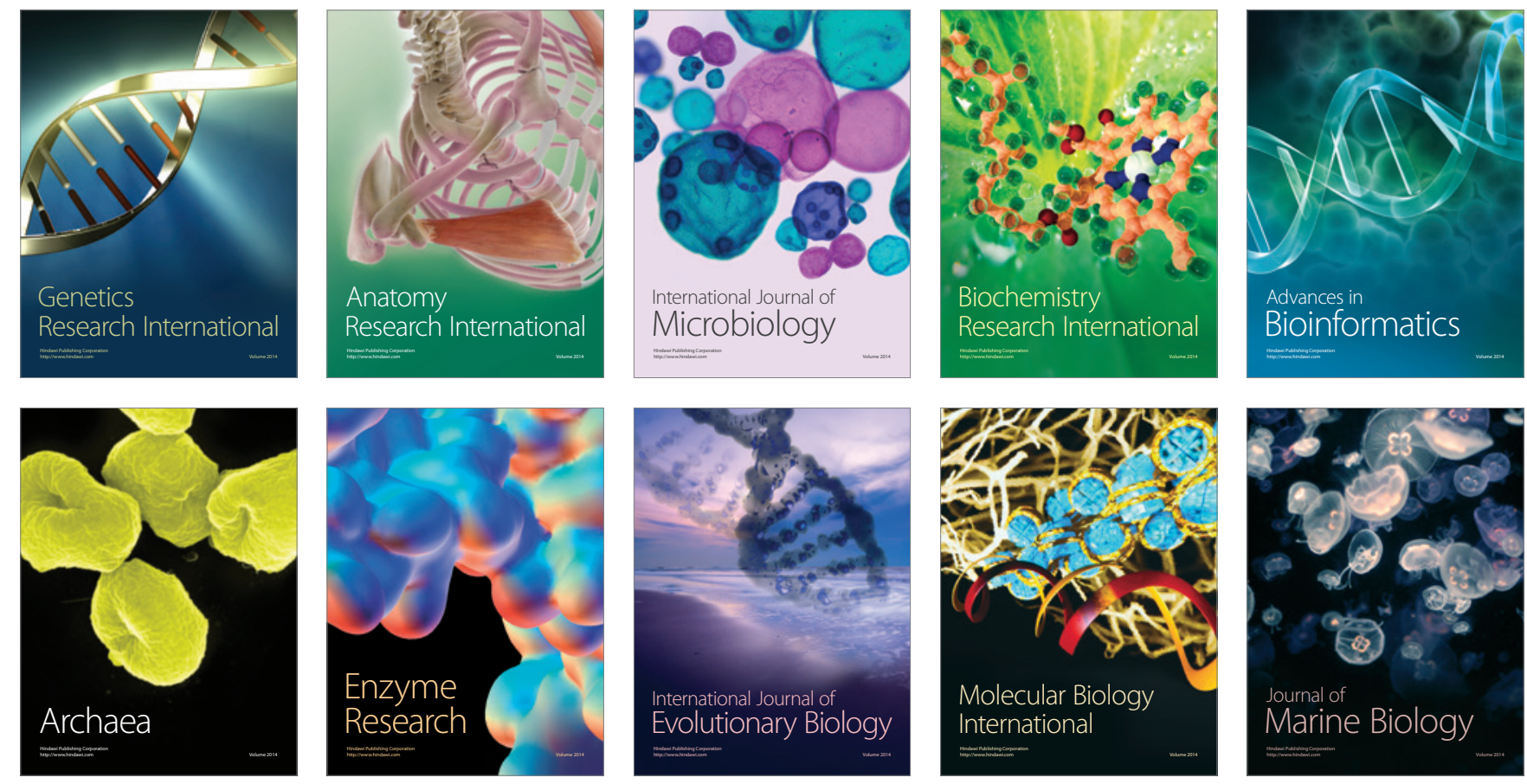